Check for updates

Cite this: RSC Adv., 2018, 8, 31255

Received 3rd July 2018

Accepted 21st August 2018

DOI: 10.1039/c8ra05691a

rsc.li/rsc-advances

\section{Tunnable rectifying performance of in-plane metal-semiconductor junctions based on passivated zigzag phosphorene nanoribbons}

\author{
ShaoLong Su, ${ }^{a}$ Jian Gong ${ }^{* a}$ and Zhi-Qiang Fan (iD *b
}

\begin{abstract}
Using first principles density functional theory, we perform a systematic study of the band structures of passivated zigzag phosphorene nanoribbons (ZPNRs) and the transport properties of in-plane metalsemiconductor junctions. It is found that the ZPNR passivated by $\mathrm{H}, \mathrm{Cl}$ or $\mathrm{F}$ atoms is a semiconductor, and the ZPNR passivated by $\mathrm{C}, \mathrm{O}$ or $\mathrm{S}$ atoms is a metal. Therefore, ZPNRs with different passivated atoms can be fabricated into an in-plane metal-semiconductor junction. The calculated current-voltage characteristics indicate that these in-plane metal-semiconductor junctions can exhibit excellent rectification behavior. More importantly, we find that the type of passivated atom plays a very important role in the rectification ratio of this in-plane metal-semiconductor junction. The findings are very useful for the further design of functional nanodevices based on ZPNRs.
\end{abstract}

\section{Introduction}

Two-dimensional and monolayered materials have attracted increasing attention day by day and are considered as an alternative to traditional bulk semiconductors for future generation nanodevices due to their intriguing physical properties. Graphene, as a monolayer of hexagonally arranged carbon atoms, is generally believed to be the first two-dimensional material and was deposited by mechanically exfoliating thin sheets from graphite in 2004 . $^{1}$ So far, experimental and theoretical research results show that graphene has extremely high mobility, high thermal conductivity, great electromechanical modulation, and excellent stiffness..$^{2-5}$ However, the absence of an intrinsic band gap imposes severe challenges on its implementation in logic and memory devices. ${ }^{6}$ Fortunately, one-dimensional graphene nanoribbons (GNRs) have tunable band gaps depending on different widths, different edge geometrical shapes, and different edge passivated atoms. Consequently, a variety of novel nonlinear electronic transport properties, such as rectification, ${ }^{7-10}$ negative differential resistance (NDR), ${ }^{11-14}$ switching, ${ }^{15-18}$ and spintronic functions have been found in nanodevices based on graphene nanoribbons. ${ }^{19-22}$

Recently, several new two-dimensional materials with puckered honeycomb structures have been found in experiment. ${ }^{23-26}$ The most prominent among them is phosphorene due to its excellent optical and electronic properties. ${ }^{27-30}$ Phosphorene has a direct band gap ranging from about $0.3 \mathrm{eV}$ (multilayer phosphorus) to

${ }^{a}$ School of Physical Science and Technology, Inner Mongolia University, Hohhot 010021, People's Republic of China.E-mail: ndgong@imu.edu.cn

${ }^{b}$ School of Physics and Electronic Science, Changsha University of Science and Technology, Changsha 410114, People's Republic of China. E-mail: zqfan@csust. edu.cn
$1.5 \mathrm{eV}$ (mono-layer phosphorene) which makes it have huge potential in applications of transistors. ${ }^{31,32}$ Like GNRs, onedimensional phosphorene nanoribbons (PNRs) also have tunable band gaps depending on different widths, different edge geometrical shapes, and different edge passivated atoms. The bare armchair phosphorene nanoribbon (APNR) is a semiconductor with an indirect band gap, and the bare zigzag phosphorene nanoribbon (ZPNR) is a metal. ${ }^{33-35}$ Through edge hydrogenation, both APNRs and ZPNRs have become semiconductors with a direct band gap. ${ }^{36,37}$ Besides hydrogen atoms, other passivated atoms also have been proved to play an important role in tuning the band gap of PNRs. ${ }^{38,39}$ However, the investigations of electronic structure and transport about nanodevices based on PNRs with different passivated atoms are not studied completely yet. In this paper, we perform a detailed analysis of a monolayer contact structure based on zigzag phosphorene nanoribbon with different passivated atoms. We find that a ZPNR passivated by $\mathrm{H}, \mathrm{Cl}$ or $\mathrm{F}$ atoms is semiconductor, and a ZPNR passivated by $\mathrm{C}, \mathrm{O}$ or $\mathrm{S}$ atoms is metal. Therefore, one can use ZPNR with different passivated atoms to fabricate an in-plane metal-semiconductor junction and to achieve the rectify behavior. The calculated results show that the type of passivated atom plays a very important role in the rectification ratio (RR) of this ZPNR in-plane metal-semiconductor junction. Our findings are very useful for the further design of functional nanodevices based on ZPNR.

\section{Methods}

Band structures of passivated ZPNRs and transport properties of in-plane metal-semiconductor junctions in this work are carried out by using Atomistix ToolKit (ATK) software package, which is based on density-functional theory in combination 
with the non-equilibrium Green's function. ${ }^{40}$ The PerdewBurke-Ernzerhof (PBE) exchange correlation functional and the generalized gradient approximation (GGA) were employed in the first-principle calculation. The real space grid techniques are used with the energy cutoff of $200 \mathrm{Ry}$ in numerical integration. The $k$-point samplings for calculations of bulk's electronic structures and electronic transport properties of ZPNR inplane metal-semiconductor junction are $1 \times 1 \times 21$ and $1 \times 1$ $\times 100$ in the $X, Y$, and $Z$ directions, respectively. A vacuum buffer space of at least $12 \AA$ is set to avoid spurious interactions. The geometries are optimized until all residual force on each atom is less than $0.01 \mathrm{eV} \AA^{-1}$. When a bias voltage is applied, the current can be calculated by the Landauer formula: $I\left(V_{\mathrm{b}}\right)=\frac{2 e}{h} \int T\left(E, V_{\mathrm{b}}\right)\left[f_{\mathrm{L}}\left(E, V_{\mathrm{b}}\right)-f_{\mathrm{R}}\left(E, V_{\mathrm{b}}\right)\right] \mathrm{d} E .^{\mathbf{4 1 , 4 2}}$ Here, $V_{\mathrm{b}}$ is the bias voltage, $T\left(E, V_{\mathrm{b}}\right)$ is the transmission coefficient, $f_{\mathrm{L}}\left(E, V_{\mathrm{b}}\right)$ and $f_{\mathrm{R}}\left(E, V_{\mathrm{b}}\right)$ are the Fermi-Dirac distribution functions of the left and right electrodes.

\section{Results and discussions}

Geometrical structures and electronic band structures of ZPNRs with different passivated atoms are shown in Fig. 1. The width of ZPNR is 16 in all six unit cells. Previous studies indicated the bare ZPNR is a metal. However, the calculated band structures in Fig. 1(a)-(c) show the bare ZPNRs turns to a semiconductor, when its two edges are passivated by $\mathrm{H}, \mathrm{F}$ or $\mathrm{Cl}$ atoms separately. The conduction band minimum (CBM) and the valence band maximum (VBM) in Fig. 1(a)-(c) all locate at the $T$ point, which gives the direct band gap. The direct band gap of H-passivated ZPNR is $1.62 \mathrm{eV}$ which is largest in three models. The direct band gap of Cl-passivated and F-passivated ZPNRs is $1.37 \mathrm{eV}$ and $1.26 \mathrm{eV}$, respectively. When two edges of the bare ZPNRs are passivated by $\mathrm{O}, \mathrm{S}$ or $\mathrm{C}$ atoms separately, the calculated band structures in Fig. 1(d)-(f) show metallic behavior. For Opassivated ZPNR, there are two bands being past the Fermi level. For S-passivated ZPNR, there are four bands crossing the Fermi level. For C-passivated ZPNR, there are two bands being past the Fermi level directly and another two bands around the Fermi level.

Besides band structures, the spatial distribution of Bloch states on near-band-edge states also plays an important role in the electronic transport properties of materials. So, the nearband-edge states 1-16 of six passivated ZPNRs (labeled in Fig. 1) were explored and their spatial distributions of Bloch states are shown in Fig. 2. For H-passivated ZPNR, the contours of Bloch state indicate that both CBM (state 1) and VBM (state 2) are contributed by $\mathrm{P}$ atoms in the central nanoribbon. ${ }^{37,43}$ The $\mathrm{H}$ atom and its connected $\mathrm{P}$ atom are nearly bare in these two states. For F-passivated and Cl-passivated ZPNRs, the contours of Bloch state indicate that both CBM (state 3 or 5) and VBM (state 4 or 6) are contributed by non-edge $\mathrm{P}$ atoms and passivated atoms jointly. For O-passivated ZPNR, the contours of Bloch states 7 and 8 are contributed by $\mathrm{O}$ atoms and edge $\mathrm{P}$ atoms. $\mathrm{P}$ atoms in the central nanoribbon have less contribution on the Bloch states. For S-passivated ZPNR, the contours of Bloch states 9 and 10 mainly delocalized on top or down edge $S$ atom, respectively. That means these two bands are mainly

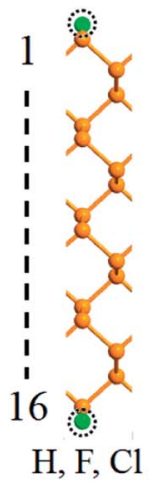

(a) 2
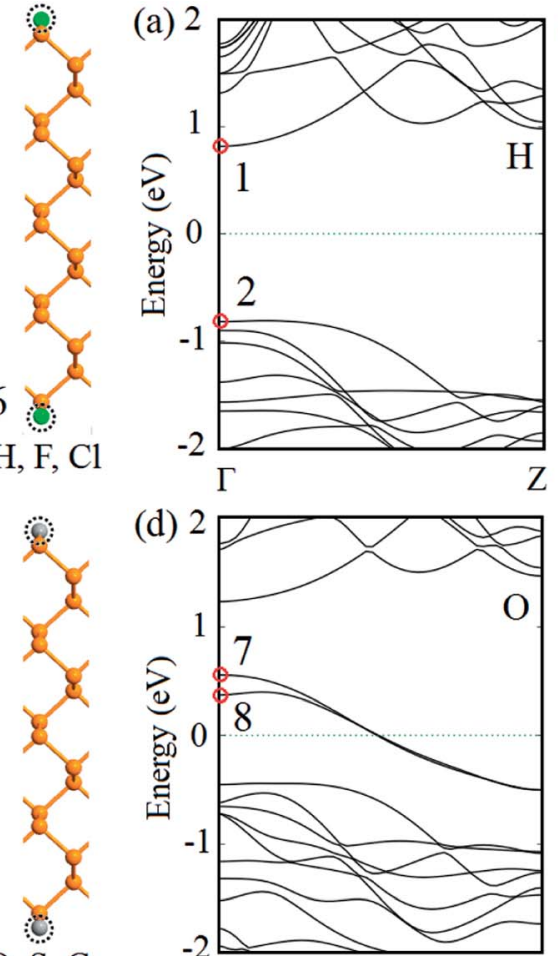

(d)

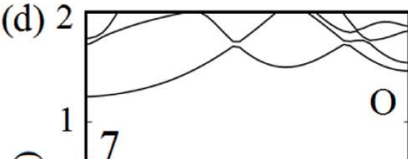

$\mathrm{O}, \mathrm{S}, \mathrm{C}$ (b)

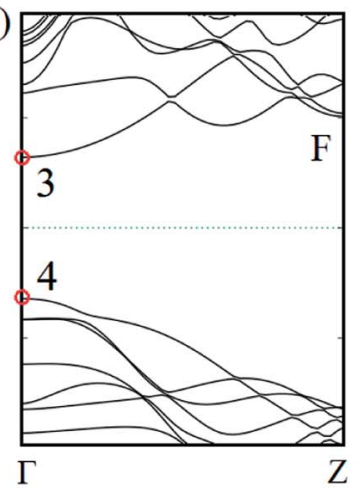

(e)

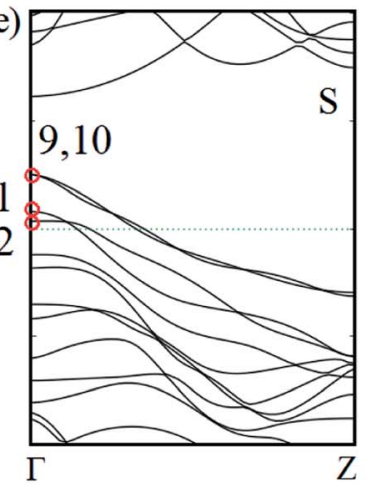

(c)

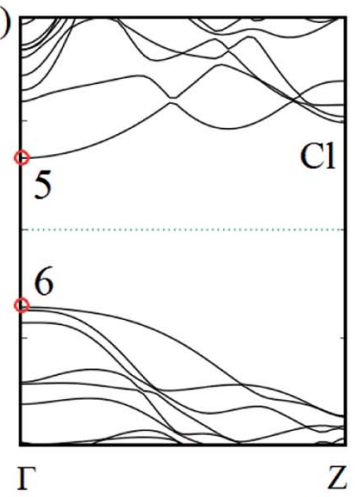

(f)

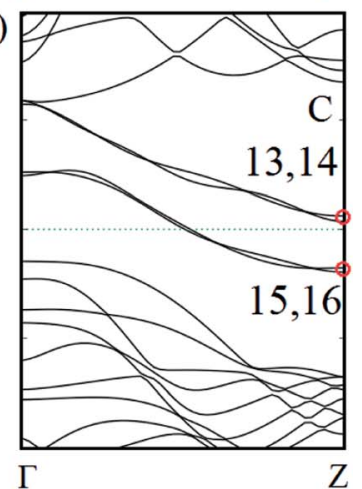

Fig. 1 Geometrical structures and electronic band structures of ZPNRs with passivated $\mathrm{H}, \mathrm{F}, \mathrm{Cl}, \mathrm{O}, \mathrm{S}$ and $\mathrm{C}$ atoms, respectively. Fermi level is set to zero. 

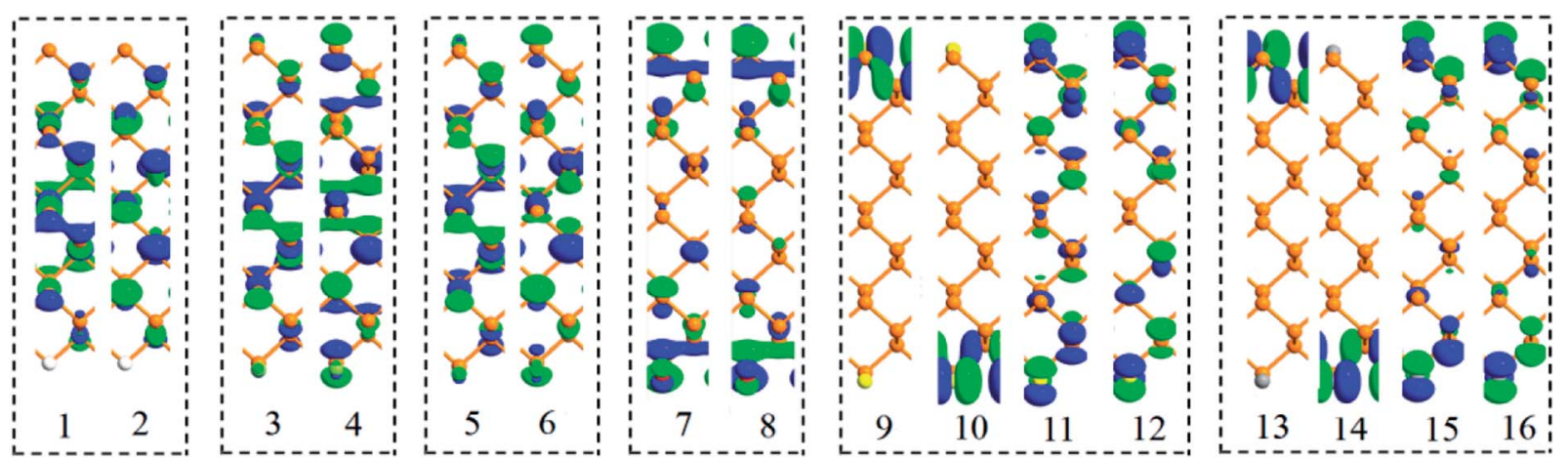

Fig. 2 Spatial distributions of Bloch states corresponding to near-band-edge states $1-16$. The isovalues are fixed 0.2 for all Bloch states.

induced by $\mathrm{S}$ atom. One can see that Bloch states 11 and 12 are contributed by $\mathrm{P}$ atoms and $\mathrm{S}$ atoms jointly. The spatial distributions of Bloch states in the C-passivated ZPNR are similar to those of S-passivated ZPNR. The Bloch states 13 and 14 mainly delocalized on top or down edge $\mathrm{C}$ atom, and the Bloch states 15 and 16 are contributed by edge $\mathrm{P}$ atoms and $\mathrm{C}$ atoms jointly.

The proposed in-plane metal-semiconductor junction based on different passivated ZPNRs are shown in Fig. 3. We divide the device into three regions: left electrode, right electrode, and central scattering region. The left electrode contains two units of $\mathrm{O}-$ passivated or C-passivated ZPNRs, and the right electrode contains two units of H-passivated, F-passivated or Cl-passivated ZPNRs. The central scattering region contains eight units of Opassivated or C-passivated ZPNRs, and eight units of $\mathrm{H}^{-}$ passivated, F-passivated or Cl-passivated ZPNRs. For simplicity, the in-plane metal-semiconductor junction based on O-passivated and $\mathrm{Cl}$-passivated $\mathrm{ZPNRs}$ is named $\mathrm{O}-\mathrm{Cl}$, and other five junctions are named $\mathrm{O}-\mathrm{H}, \mathrm{O}-\mathrm{F}, \mathrm{C}-\mathrm{Cl}, \mathrm{C}-\mathrm{H}$ and $\mathrm{C}-\mathrm{F}$, respectively.

The transmission spectra of these six in-plane metal-semiconductor junctions are shown in Fig. 4. In Fig. 4(a), there are large transmission forbidden regions because of the large band gaps of Cl-passivated, H-passivated and F-passivated ZPNRs. The ranges of transmission forbidden regions of $\mathrm{O}-\mathrm{Cl}$ and $\mathrm{O}-\mathrm{H}$ junctions are similar to each other. However, the transmission coefficients of the $\mathrm{O}-\mathrm{H}$ junction around $-0.75 \mathrm{eV}$ are smaller than that of the $\mathrm{O}-\mathrm{Cl}$ junction. It can be explained by the different spatial distributions of Bloch states on the VBM of $\mathrm{H}^{-}$ passivated and Cl-passivated ZPNRs. The range of transmission forbidden region of the $\mathrm{O}-\mathrm{F}$ junction is shorter than other two junctions due to the smaller band gap of F-passivated ZPNR. Although the band gaps of Cl-passivated, $\mathrm{H}$-passivated and Fpassivated ZPNRs are different, the right edges of transmission forbidden regions all locate at $1.23 \mathrm{eV}$ for three junctions. In addition, one can find that the ranges of transmission forbidden regions are actually larger than the corresponding band gaps of Cl-passivated, H-passivated and F-passivated ZPNRs. In Fig. 4(b), the transmission spectra are similar to that of Fig. 4(a). The range of the transmission forbidden region in the $\mathrm{C}-\mathrm{F}$ junction is shorter than other two junctions. However, the right edge of transmission forbidden regions all locate at $0.97 \mathrm{eV}$ for $\mathrm{C}-\mathrm{Cl}, \mathrm{C}-\mathrm{H}$ and $\mathrm{C}-\mathrm{F}$ junctions. Therefore, the ranges of the transmission forbidden regions in Fig. 4(b) are shorter than that of Fig. 4(a).

To explain the right edges of transmission forbidden regions all locating at the same energy in Fig. 4(a), we plot the projected density of states of the left electrode and the right electrode for $\mathrm{O}-\mathrm{Cl}, \mathrm{O}-\mathrm{H}$ and $\mathrm{O}-\mathrm{F}$ junctions in Fig. 5. Here, $\mathrm{L}-\mathrm{O}$ indicates projected density of states of the left O-passivated ZPNR and R$\mathrm{Cl}, \mathrm{R}-\mathrm{H}$ and $\mathrm{R}-\mathrm{F}$ indicate projected density of states of the right Cl-passivated, H-passivated and F-passivated ZPNRs, respectively. In Fig. 5(a), one can find that a forbidden region exists in the projected density of states of the right Cl-passivated ZPNR. The range of this forbidden region is equal to the band gap of the Cl-passivated ZPNR. For the left O-passivated ZPNR, a series

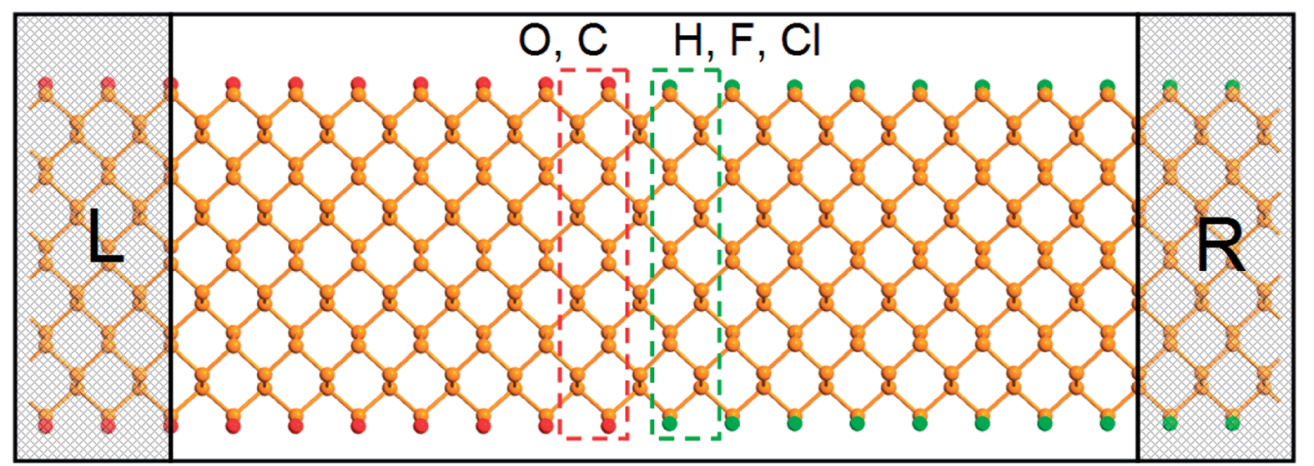

Fig. 3 Geometry schematic illustration of the proposed metal-semiconductor junction based on different passivated ZPNRs. The left (L) and right $(R)$ electrodes are indicated by rectangular frames. 


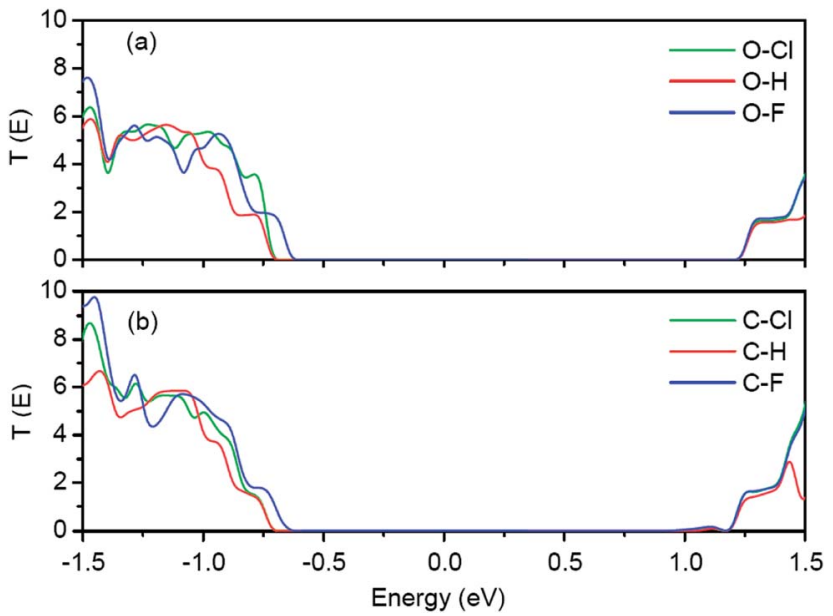

Fig. 4 (a) Transmission spectra of $\mathrm{O}-\mathrm{Cl}, \mathrm{O}-\mathrm{H}$ and $\mathrm{O}-\mathrm{F}$ junctions. (b) Transmission spectra of $\mathrm{C}-\mathrm{Cl}, \mathrm{C}-\mathrm{H}$ and $\mathrm{C}-\mathrm{F}$ junctions. Fermi energy is set to zero in the energy scale.

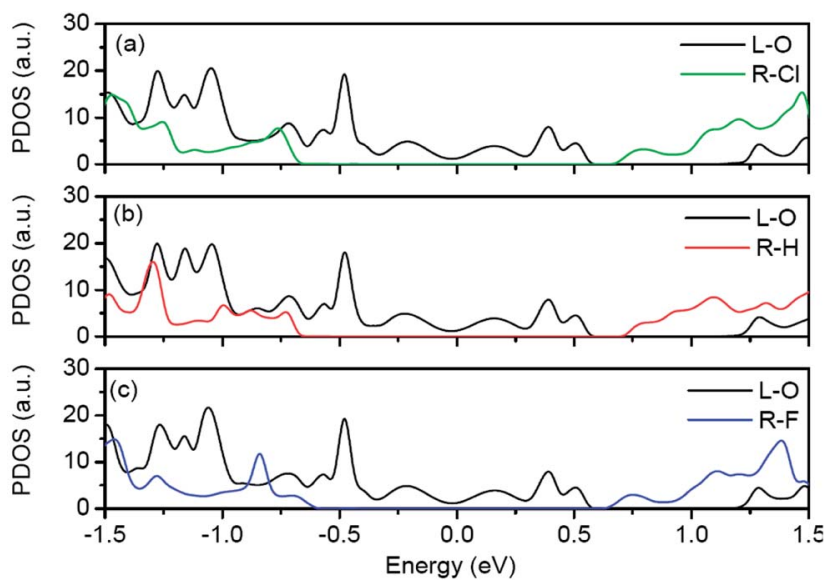

Fig. 5 Projected density of states of left electrode and right electrode for $\mathrm{O}-\mathrm{Cl}, \mathrm{O}-\mathrm{H}$ and $\mathrm{O}-\mathrm{F}$ junctions, respectively. Fermi energy is set to zero in the energy scale. of peaks spread around Fermi energy. But, there still exists a forbidden region from $0.56 \mathrm{eV}$ to $1.23 \mathrm{eV}$, which is in agreement with band structure of the O-passivated ZPNR in Fig. 1(d). As a result, the right edge of transmission forbidden region of the $\mathrm{O}-\mathrm{Cl}$ junction locates at $1.23 \mathrm{eV}$ and the range of transmission forbidden region is actually larger than the corresponding band gap of the Cl-passivated ZPNR. When the passivated atoms of right ZPNR change from $\mathrm{Cl}$ atom to $\mathrm{H}$ or $\mathrm{F}$ atoms, the projected density of state of the left O-passivated ZPNR in Fig. 5(b) or (c) is nearly same to that in Fig. 5(a). Consequently, the right edge of transmission forbidden region of the $\mathrm{O}-\mathrm{H}$ junction or the $\mathrm{O}-\mathrm{F}$ junction also locate at $1.23 \mathrm{eV}$.

The current-voltage characteristics of six in-plane metalsemiconductor junctions are illustrated in Fig. 6. In Fig. 6(a), one can find the currents of $\mathrm{O}-\mathrm{H}$ and $\mathrm{O}-\mathrm{F}$ junctions are always small in the negative bias region. However, the currents of these two junctions will increase rapidly after $0.25 \mathrm{~V}$. So, the currentvoltage curves in the whole bias region are asymmetrical and show the rectification performances. For the $\mathrm{O}-\mathrm{Cl}$ junction, the current-voltage curve is also asymmetrical. But, the rectification performance is not intensive because its currents are also big after $-0.3 \mathrm{~V}$. In general, the rectification performance can be evaluated by the rectification ratio which is the ratio of the positive bias current to the negative bias one, RR $(\mathrm{V})=I(+\mathrm{V}) /$ $I(-\mathrm{V})$. For the $\mathrm{O}-\mathrm{F}$ junction, $\mathrm{RR}$ will increase gradually with the bias, and the maximal RR nearly approximates $10^{4}$ at $0.35 \mathrm{~V}$. The average RR of the $\mathrm{O}-\mathrm{H}$ junction is smaller than that of the $\mathrm{O}-\mathrm{F}$ junction. The maximal RR can approximate $10^{3}$ at $0.4 \mathrm{~V}$. The average $\mathrm{RR}$ of the $\mathrm{O}-\mathrm{Cl}$ junction is very small because of its large current at negative bias region. In Fig. 6(b), one can find that currents of $\mathrm{C}-\mathrm{Cl}, \mathrm{C}-\mathrm{H}$ and $\mathrm{C}-\mathrm{F}$ junctions are always very small in the negative bias region. However, currents of the $\mathrm{C}-\mathrm{F}$ junction will increase rapidly after $0.25 \mathrm{~V}$ and the currents of C$\mathrm{Cl}$ and $\mathrm{C}-\mathrm{H}$ junctions will increase rapidly after $0.3 \mathrm{~V}$. Consequently, current-voltage characteristics of these three junctions also show the rectification performances. The average RR of C-F junction is also largest in three junctions. In addition, the RRs of $\mathrm{C}-\mathrm{Cl}, \mathrm{C}-\mathrm{H}$ and $\mathrm{C}-\mathrm{F}$ junctions are all larger than those of $\mathrm{O}-\mathrm{Cl}$,
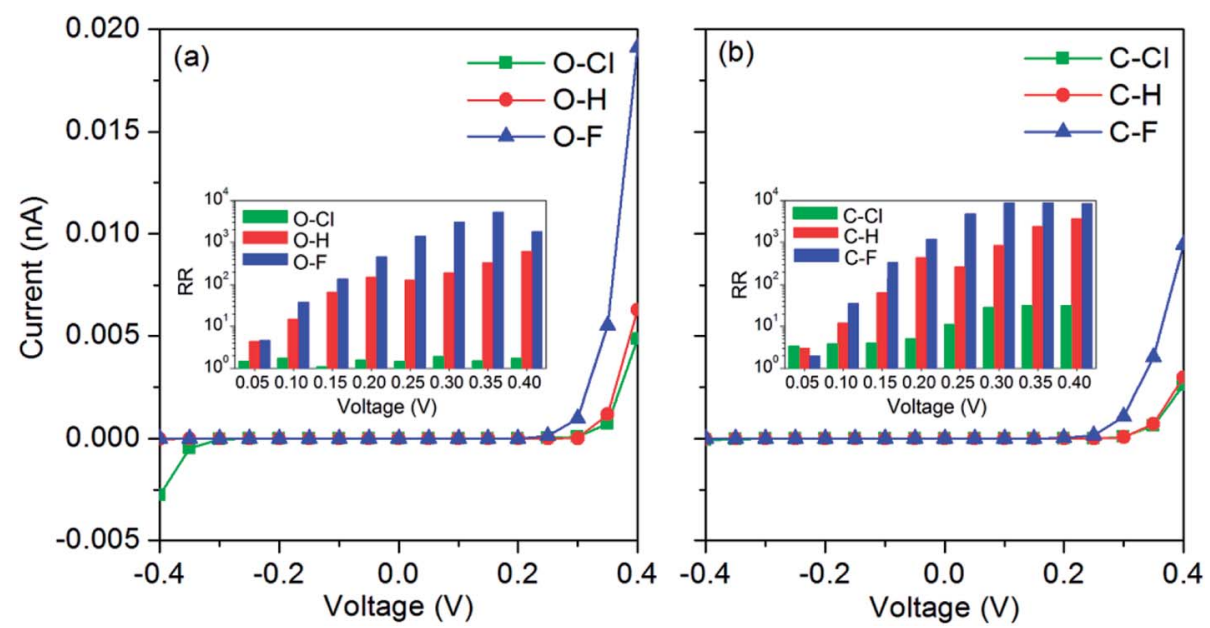

Fig. 6 (a) Current-voltage characteristics of $\mathrm{O}-\mathrm{Cl}, \mathrm{O}-\mathrm{H}$ and $\mathrm{O}-\mathrm{F}$ junctions. (b) Current-voltage characteristics of $\mathrm{C}-\mathrm{Cl}$, $\mathrm{C}-\mathrm{H}$ and $\mathrm{C}-\mathrm{F}$ junctions. The each inset figure is corresponding rectification ratio RR of in-plane metal-semiconductor junction. 


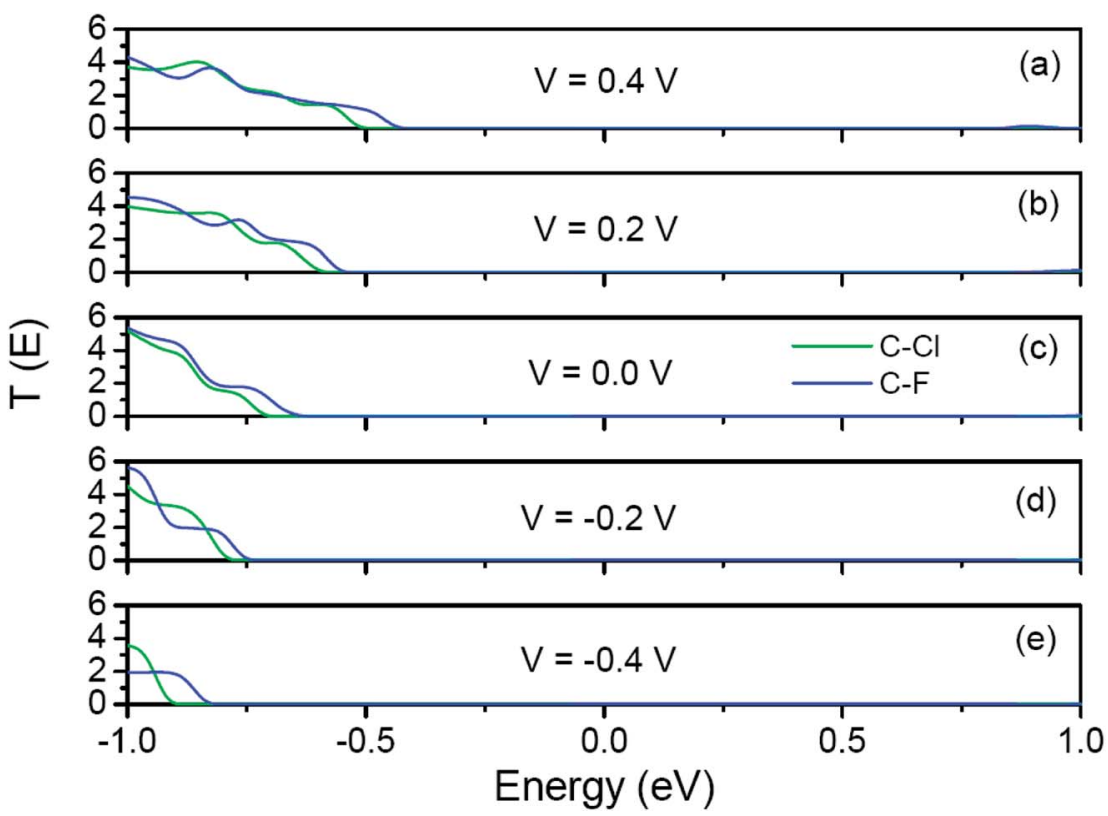

Fig. 7 Transmission spectra of $\mathrm{C}-\mathrm{Cl}$ and $\mathrm{C}-\mathrm{F}$ junctions at several representative bias voltages. Fermi energy is set to zero in the energy scale.

$\mathrm{O}-\mathrm{H}$ and $\mathrm{O}-\mathrm{F}$ junctions, respectively. Therefore, one can conclude that the types of the passivated atoms play an important role on the rectification performances of these inplane metal-semiconductor junctions.

To reveal the origins of the different rectification performances in Fig. 6, we plot the transmission spectra of $\mathrm{C}-\mathrm{Cl}$ and $\mathrm{C}-\mathrm{F}$ junctions at several representative bias voltages in Fig. 7. As we describing in the method, the current of the junctions is calculated by the Landauer formula: $I\left(V_{\mathrm{b}}\right)=\frac{2 e}{h} \int T\left(E, V_{\mathrm{b}}\right)\left[f_{\mathrm{L}}\left(E, V_{\mathrm{b}}\right)-f_{\mathrm{R}}\left(E, V_{\mathrm{b}}\right)\right] \mathrm{d} E$. Here, $V_{\mathrm{b}}$ is the bias voltage, $T\left(E, V_{\mathrm{b}}\right)$ is the transmission coefficient. When two junctions are applied on the positive bias, the potential of left electrode is raised and the potential of right electrode is reduced. So, the band gap of right Cl-passivated or F-passivated ZPNR shifts to right relative to Fermi energy (left C-passivated ZPNR). As a result, the transmission spectra would shift to right gradually and the left edges of the transmission forbidden regions get close to Fermi energy. So, the total transmission coefficients in the bias window increase gradually leading to the increasing of the current. However, in negative bias region, the potential of left electrode is reduced and the potential of right electrode is raised. So, the transmission spectra would shift to left with increasing of negative bias. The left edges of the transmission forbidden regions get far away from Fermi energy. Changes of total transmission coefficients in the bias window are quite inconspicuous resulting in the small current. As a result, current-voltage characteristics of two junctions show the rectification performances. Because the F-passivated ZPNR has a smaller band gap than the Cl-passivated ZPNR, the left edge of the transmission forbidden region of the $\mathrm{C}-\mathrm{F}$ junction is closer to Fermi energy than that of the $\mathrm{C}-\mathrm{Cl}$ junction. At the same positive bias, the current of the $\mathrm{C}-\mathrm{F}$ junction is larger than that of $\mathrm{C}-\mathrm{Cl}$ junction. That's why we can find that the rectification performance of the $\mathrm{C}-\mathrm{F}$ junction is more intensive than that of the $\mathrm{C}-\mathrm{Cl}$ junction.

\section{Conclusion}

In summary, we have investigated the band structures of passivated ZPNRs and transport properties of in-plane metalsemiconductor junctions by using density-functional theory in combination with the non-equilibrium Green's function. Our study shows that the ZPNR passivated by $\mathrm{H}, \mathrm{Cl}$ or $\mathrm{F}$ atoms is a semiconductor, and the ZPNR passivated by $\mathrm{C}, \mathrm{O}$ or $\mathrm{S}$ atoms is a metal. Then, we use ZPNRs with different passivated atoms to fabricate an in-plane metal-semiconductor junction. The calculated current-voltage characteristics indicate that these inplane metal-semiconductor junctions can exhibit excellent rectification behavior. More importantly, we find that the type of passivated atom plays a very important role in the rectification ratio (RR) of this in-plane metal-semiconductor junction. The maximal RR of $\mathrm{O}-\mathrm{F}$ and $\mathrm{C}-\mathrm{F}$ junctions nearly approximates $10^{4}$. The findings are very useful for the further design of functional nanodevices based on ZPNR.

\section{Conflicts of interest}

There are no conflicts to declare.

\section{Acknowledgements}

This work was supported by the National Natural Science Foundation of China (Grant No. 11464032 and 11674039). One of authors Zhi-Qiang Fan also express his thanks for the Scientific Research Fund of Hunan Provincial Education Department (Grant No. 15A004). 


\section{References}

1 K. S. Novoselov, A. K. Geim, S. V. Morozov, D. Jiang, Y. Zhang, S. V. Dubonos, I. V. Grigorieva and A. A. Firsov, Science, 2004, 306, 666.

2 E. H. Hwang, S. Adam and S. D. Sarma, Phys. Rev. Lett., 2007, 98, 186806.

3 S. Ghosh, I. Calizo, D. Teweldebrhan, E. P. Pokatilov, D. L. Nika, A. A. Balandin, W. Bao, F. Miao and C. N. Lau, Appl. Phys. Lett., 2008, 92, 151911.

4 J. S. Bunch, A. M. van der Zande, S. S. Verbridge, I. W. Frank, D. M. Tanenbaum, J. M. Parpia, H. G. Craighead and P. L. McEuen, Science, 2008, 315, 490.

5 X. J. Wu and X. C. Zeng, Nano Lett., 2009, 9, 250.

6 F. Schwierz, Nat. Nanotechnol., 2010, 5, 487.

7 M. G. Zeng, L. Shen, M. Yang, C. Zhang and Y. P. Feng, Appl. Phys. Lett., 2011, 98, 053101.

8 J. Li, Z. H. Zhang, G. Kwong, W. Tian, Z. Q. Fan and X. Q. Deng, Carbon, 2013, 61, 284.

9 H. Q. Wan, B. H. Zhou, W. H. Liao and G. H. Zhou, J. Chem. Phys., 2013, 138, 034705.

10 D. Zhang, M. Q. Long, X. J. Zhang, J. Ouyang and H. Xu, J. Appl. Phys., 2017, 121, 093903.

11 Z. Y. Li, H. Y. Qian, J. Wu, B. L. Gu and W. H. Duan, Phys. Rev. Lett., 2008, 100, 206802.

12 K. M. M. Habib and R. K. Lake, Phys. Rev. B, 2012, 86, 045418. 13 S. L. Yan, M. Q. Long, X. J. Zhang and H. Xu, Phys. Lett. A, 2014, 378, 960.

14 J. Liu, Z. H. Zhang, X. Q. Deng, Z. Q. Fan and G. P. Tang, Org. Electron., 2015, 18, 135.

15 X. R. Wang, X. L. Li, L. Zhang, Y. K. Yoon, P. K. Weber, H. L. Wang, J. Guo and H. J. Dai, Science, 2009, 324, 768.

16 H. Q. Wan, B. H. Zhou, X. W. Chen, C. Q. Sun and G. H. Zhou, J. Phys. Chem. C, 2012, 116, 2570.

17 Z. Q. Fan and K. Q. Chen, Sci. Rep., 2014, 4, 5976.

18 Z. Q. Fan, Z. H. Zhang, F. Xie, X. Q. Deng, G. P. Tang, C. H. Yang and K. Q. Chen, Org. Electron., 2015, 18, 101.

19 D. Wang, Z. H. Zhang, Z. Zhu and B. Liang, Sci. Rep., 2014, 4, 7587.

20 Z. Q. Fan, F. Xie, X. W. Jiang, Z. M. Wei and S. S. Li, Carbon, 2016, 98, 179.

21 L. L. Cui, M. Q. Long, X. J. Zhang, X. M. Li, D. Zhang and B. C. Yang, Phys. Lett. A, 2016, 380, 730.
22 Z. Q. Fan, W. Y. Sun, Z. H. Zhang, X. Q. Deng, G. P. Tang and H. Q. Xie, Carbon, 2017, 122, 687.

23 N. Lu, H. Y. Guo, L. Li, J. Dai, L. Wang, W. N. Mei, X. J. Wu and X. C. Zeng, Nanoscale, 2014, 6, 2879.

24 B. Zhu, X. J. Zhang, B. W. Zeng, M. J. Li and M. Q. Long, Org. Electron., 2017, 49, 45.

25 Z. Q. Fan, X. W. Jiang, J. W. Luo, L. Y. Jiao, R. Huang, S. S. Li and L. W. Wang, Phys. Rev. B, 2017, 96, 165402.

26 Z. Q. Fan, X. W. Jiang, J. Z. Chen and J. W. Luo, ACS Appl. Mater. Interfaces, 2018, 10, 19271.

27 L. Li, Y. Yu, G. J. Ye, Q. Ge, X. Ou, H. Wu, D. Feng, X. H. Chen and Y. Zhang, Nat. Nanotechnol., 2014, 9, 372.

28 Q. Wei and X. Peng, Appl. Phys. Lett., 2014, 104, 251915.

29 X. L. Li, L. Ma, D. Y. Wang, X. C. Zeng, X. J. Wu and J. L. Yang, Nanoscale, 2016, 8, 17801.

30 Y. Jing, X. Zhang and Z. Zhou, Wiley Interdiscip. Rev.: Comput. Mol. Sci., 2016, 6, 5.

31 A. S. Rodin, A. Carvalho and A. H. C. Neto, Phys. Rev. Lett., 2014, 112, 176801.

32 S. Das, W. Zhang, M. Demarteau, A. Hoffmann, M. Dubey and A. K. Roelofs, Nano Lett., 2014, 14, 5733.

33 H. Y. Guo, N. Lu, J. Dai, X. J. Wu and X. C. Zeng, J. Phys. Chem. C, 2014, 118, 14051.

34 W. F. Li, G. Zhang and Y. W. Zhang, J. Phys. Chem. C, 2014, 118, 22368.

35 A. Carvalho, A. S. Rodin and A. H. Castro Neto, Europhys. Lett., 2014, 108, 47005.

36 V. Tran and L. Yang, Phys. Rev. B, 2014, 89, 245407.

37 Q. Y. Wu, L. Shen, M. Yang, Y. Q. Cai, Z. G. Huang and Y. P. Feng, Phys. Rev. B, 2015, 92, 035436.

38 X. H. Peng, A. Copple and Q. Wei, J. Appl. Phys., 2014, 116, 144301.

39 N. Chen, Y. P. Wang, Y. W. Mu, Y. F. Fan and S. D. Li, Phys. Chem. Chem. Phys., 2017, 19, 25441.

40 M. Brandbyge, J. L. Mozos, P. Ordejón, J. Taylor and K. Stokbro, Phys. Rev. B, 2002, 65, 165401.

41 M. Büttiker, Y. Imry, R. Landauer and S. Pinhas, Phys. Rev. B, 1985, 31, 6207.

42 Z. Q. Fan, K. Q. Chen, Q. Wan, B. S. Zhou, W. H. Duan and Z. Shuai, Appl. Phys. Lett., 2008, 92, 263304.

43 Z. Q. Fan, W. Y. Sun, X. W. Jiang, J. W. Luo and S. S. Li, Org. Electron., 2017, 44, 20. 